\title{
Human Mars Entry, Descent, and Landing Architecture Study Overview
}

\author{
Alicia D. Cianciolo ${ }^{1}$ \\ NASA Langley Research Center, Hampton, Virginia, 23681 \\ and \\ Tara T. Polsgrove ${ }^{2}$ \\ NASA Marshall Space Flight Center, Huntsville, Alabama 35812
}

\begin{abstract}
The Entry, Descent, and Landing (EDL) Architecture Study is a multi-NASA center activity to analyze candidate EDL systems as they apply to human Mars landing in the context of the Evolvable Mars Campaign. The study, led by the Space Technology Mission Directorate (STMD), is performed in conjunction with the NASA's Science Mission Directorate and the Human Architecture Team, sponsored by NASA's Human Exploration and Operations Mission Directorate. The primary objective is to prioritize future STMD EDL technology investments by (1) generating Phase A-level designs for selected concepts to deliver $20 \mathrm{t}$ human class payloads, (2) developing a parameterized mass model for each concept capable of examining payloads between 5 and $40 \mathrm{t}$, and (3) evaluating integrated system performance using trajectory simulations. This paper summarizes the initial study results.
\end{abstract}

\section{Introduction}

L ANDING humans on Mars will require entry, descent, and landing (EDL) capability beyond the current state of the art. The Mars Science Laboratory (MSL) mission successfully delivered just less than one metric ton to the surface of mars. Human Mars missions will require multiple landings, each delivering nearly twenty times the MSL payload. In addition, an order of magnitude improvement in precision landing capability will be necessary. In order to prioritize technology investments that will meet human landing requirements, NASA directorates have collaborated in a multi-center Entry, Descent, and Landing Architecture Study (EDLAS) to analyze candidate EDL systems. The study, which is sponsored by the Space Technology Mission Directorate (STMD), collaborates with the Human Exploration and Operations Mission Directorate's (HEOMD) Human Architecture Team (HAT) to define human mission architecture constraints. The Science Mission Directorate provides robotic EDL mission heritage and evolutionary science payload options that may offer opportunities for human Mars precursor demonstrations.

The NASA Human Architecture Team is currently studying an Evolvable Mars Campaign (EMC) ${ }^{1}$, a space exploration framework leading to a set of evolutionary paths from the current spaceflight infrastructure and capabilities to the ultimate goal of landing people on the surface of Mars and returning them to Earth. General EMC assumptions that pertain to this study include launching on a Space Launch System (SLS) with a 10 m payload fairing and using a Solar Electric Propulsion (SEP) system for in-space transportation. The SEP system delivers a lander carrying a 20 t payload; four payloads will be needed to support a crew of four on the surface for up to 300 days. For the purposes of this study, the Mars Ascent Vehicle (MAV) payload configuration was selected for EDL system evaluation. This payload configuration includes the MAV, shown in Figure 1, and an In-Situ Resource Utilization plant for ascent vehicle propellant production. This configuration was selected because the MAV is the single largest indivisible EMC payload

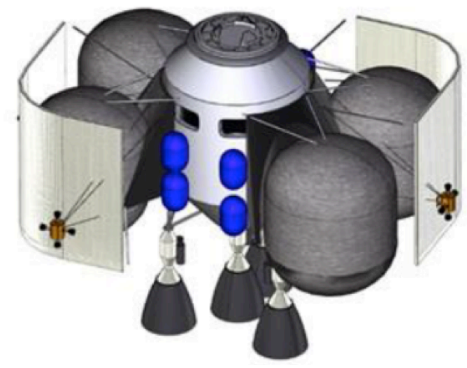

Figure 1. The Mars Ascent Vehicle.

\footnotetext{
${ }^{1}$ Aerospace Engineer, Atmospheric Flight and Entry Systems Branch, MS 489, AIAA Senior Member

${ }^{2}$ Human Architectures Team Lead, Flight Programs and Partnership Office, Mail Stop (MS) FP30 
element, as well as the most challenging to package. A full description of the current EMC MAV design is provided in reference 2 .

The EMC is currently considering two SEP options for in-space transportation. The first, called the SEPChemical option, uses an Asteroid Robotic Redirect Mission (ARRM) derived $150 \mathrm{~kW}$ class SEP for cargo missions and a chemical propellant (liquid oxygen and methane) for crew missions. In this architecture, landers carrying cargo elements are released from the SEP stage days prior to Mars arrival and use aerobraking to achieve a 1 Sol parking orbit. The second option, called Hybrid SEP, uses a $300 \mathrm{~kW}$ ARRM derived SEP paired with currently available storable chemical propulsion for crew or cargo missions. Since the Hybrid SEP option reuses the propulsion and habitat module for the trip back to Earth, it spirals only to a 5 sol highly elliptical orbit and does not require an aerocapture maneuver. Since at least one transportation option includes aerobraking, the EDL technologies under consideration must be capable of surviving both the aerocapture and EDL heating environments.

The key EMC assumptions for launch vehicle, payload and in-space transportation provided the framework, ground rules and assumptions that are used to generate the EDLAS detailed Phase A designs. Other Phase A design activities incorporated into this study include flowing the EMC project requirements down to the system and subsystem level, assessing technology requirements, evaluating and refining subsystem interfaces, completing a system-level designs, and developing a credible mission/system architecture. ${ }^{3}$

The EDLAS effort is described in the following sections. Section 2 summarizes the EDL technologies selected for the Phase A detailed designs and the motivation for their selection. Section 2 also describes how the entry technologies interface with other mission elements, specifically the launch vehicle, payload and descent stage, and describe the integrated mission architecture and concept of operations. Section 3 outlines the two-pronged study approach for developing parametric models in parallel to detailed vehicle point designs. The detailed designs, based on EMC project requirements including payload definition and launch vehicle payload fairing, assist in refining subsystem interfaces and mass estimates. The detailed designs also identify subsystem and integration challenges to address in order to complete the system level designs. The detailed designs serve as anchor points for the parametric models. Parametric models assess the technology requirements and performance over a wide range of parameters such as ballistic number and entry velocity. Section 4 summarizes the figures of merit used to compare the architectures. Finally, Section 5 provides an initial summary of the results of the analysis performed to assess the technology requirements, and lists primary finding and observations of the study.

\section{EDL Technologies and Architectures}

A recent human Mars mission architecture, the Design Reference Architecture 5 (DRA5), assumed a rigid mid lift-to-drag (L/D) vehicle $(10 \times 30 \mathrm{~m})$ for EDL to deliver a $40 \mathrm{t}$ payload to the surface of Mars. A follow-on study, the Entry, Descent, and Landing Systems Analysis (EDLSA) ${ }^{5}$ study, broadened the evaluation of EDL technologies to consider deployables and found that they saved significant mass over rigid vehicles. Concurrently, packaging efforts for EMC identified many challenges of packaging the $40 \mathrm{t}$ payload in a single launch and landing vehicle. The center of gravity location required for entry was not ideal for launch and landing. Landing and surface stability and cargo offloading were also a concern. Additionally, packaging margin and loss of an entire mission supply were major risks. Therefore, the current EMC studies assume a larger number of landers per surface mission using common descent stages to deliver smaller payloads (i.e. $20 \mathrm{t}$ instead of $40 \mathrm{t}$ ). The EMC also avoids in-space or Mars surface assembly of mission critical elements. Therefore, the MAV emerged as the largest indivisible element and was selected as the primary Mars surface payload for the study.

The payload is integrated to a descent stage. The descent stage carries all the propellant and thrusters required to control the vehicle during aerocapture and EDL. The descent stage also serves as the surface operations platform after landing. The descent stage is integrated with the entry decelerator technology, an interface that is highly technology specific. Finally, the EMC assumes that all three major EDL elements, the surface payload, descent stage, and entry technology, are integrated with the SEP stage in the $9.1 \mathrm{~m}$ dynamic envelope of the $10 \mathrm{~m}$ diameter SLS payload fairing.

EDLAS considered four entry decelerator technologies. Two are considered evolutionary technologies, meaning they are an extension of flight heritage vehicles from both human and robotic missions. The first, drawing some heritage from shuttle experience and similar to the original DRA5 design, but reduced in size to accommodate the smaller EMC payload, is a rigid Mid L/ $\mathrm{D}^{6}$ vehicle. The vehicle, modeled loosely after the Terrestrial Return Vehicle (TRV) shape, is shown in Figure 2a. The dimensions of the vehicle for this study are $8.8 \times 19.8 \mathrm{~m}$ (not including the aft body flaps) and it flies at an angle of attack of 55 degrees with an L/D of 0.54 . The second evolutionary vehicle is a capsule that draws heritage from robotic Mars missions and looks similar to a scaled up version of the Mars Science Laboratory (MSL) capsule. The vehicle flies with an L/D of 0.24, similar to MSL. See Figure 2b. Both of 
the evolutionary designs include rigid shells covering the payload for aerocapture and entry and have ballistic coefficients greater than $300 \mathrm{~kg} / \mathrm{m}^{2}$. Both will also require reconfiguration of the HAT payloads to fit the vehicle geometry.

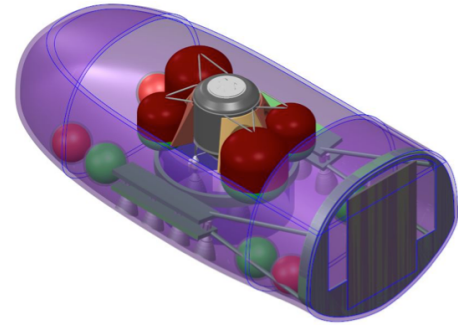

(a) Rigid Mid L/D

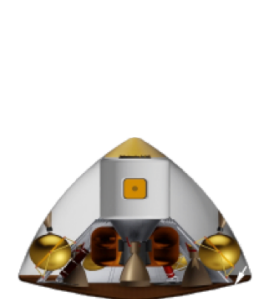

(b) Heritage Capsule

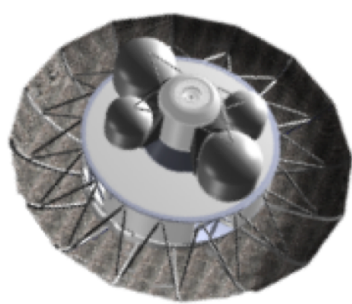

(c) ADEPT

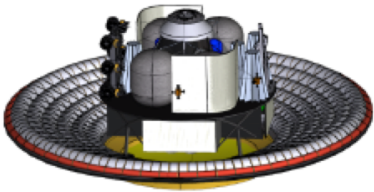

(d) HIAD

Figure 2. EMC MAV payload integrated into EDL technologies, except heritage capsule, which is shown with a different payload.

The Mid L/D vehicle has the descent stage integrated into the entry technology structure while some versions of the heritage capsule do not. If the capsule jettisons the backshell and heatshield similar to MSL, then, unlike the Mid $\mathrm{L} / \mathrm{D}$ vehicle, the descent stage would not be integrated into the rigid shell structure.

It is noted that the baseline assumptions for the capsule design ${ }^{7}$ shown here were not compatible with EMC assumptions (i.e. payload elements were not the same and the capsule assumed storable propellants while the three others assumed ISRU based LOX-methane engines). A complete assessment of the heritage capsule design using a modified backshell, more like Orion or Apollo, to accommodate the larger EMC payloads is being performed to determine if the capsule concept offers advantages over the other EDL technologies being considered. However, this work is still on going, so no analysis or results of the heritage capsule design are presented in this paper.

The remaining two entry technologies considered revolutionary rather than evolutionary, are deployable vehicles. Although deployables have not been used extensively in Mars or human missions, there have been many successful subscale test flights at Earth. The deployable technology addresses the challenge of the low atmosphere density on Mars by increasing the drag area beyond the limits of the launch vehicle shroud. Previous studies also showed deployables to save significant mass over rigid options.

The first rigid deployable is the Adaptive Deployable Entry and Placement Technology (ADEPT) ${ }^{8}$ configuration. Resembling an umbrella, ADEPT consists of a flexible 3-D woven TPS structural membrane across rib structures that are stowed during launch and deployed in space. The TPS is designed to survive both the aerocapture and entry thermal pulses. The initial ADEPT design used an asymmetric $16 \mathrm{~m}$ diameter shape that provided the desired lift performance during aerocapture and entry, rather than relying on a movable mass or aerodynamic device to produce lift by changing the angle of attack. The asymmetric ADEPT had an L/D of 0.27. An image of the asymmetric ADEPT with the EMC MAV payload and descent stage are shown in Figure 2c. However, as discussed later, difficulties with the asymmetric design led to configuration change to a symmetric 70-degree cone with an aerodynamic tab.

The second deployable technology is the Hypersonic Inflatable Aerodynamic Decelerator (HIAD) ${ }^{9}$. The HIAD design uses an inflatable structure covered by a flexible TPS. The inflatable structure is a stacked-torus design, meaning that it is built as a conical stack of pressurized rings, connected to each other and anchored to the central rigid nose by radial structural webbing. While HIAD flexible TPS samples have survived multiple heat pulse testing, the performance of the material deflated after aerocapture and reinflated up to a year later has not been characterized, and it is infeasible to carry gas generators onboard to keep the HIAD inflated during an extended loiter in Mars orbit between aerocapture and entry. Therefore, the decision was made to carry two separate HIAD's, one for aerocapture and one for entry. The aerocapture HIAD is jettisoned before the orbit circularization burn after the atmospheric pass. To maintain similar ballistic coefficients in current trajectory simulations, the aerocapture HIAD is slightly larger, $18.8 \mathrm{~m}$, compared to the entry HIAD, which has a $16.7 \mathrm{~m}$ diameter. The nominal HIAD vehicle has an $\mathrm{L} / \mathrm{D}$ of 0.2 , uses a symmetric $70 \mathrm{deg}$ cone shape, and is shown integrated with the EMC MAV payload in Figure 2d. A full description of the HIAD entry design is provided in Ref 10. The diameter of both low $\mathrm{L} / \mathrm{D}$ vehicles was selected such that the vehicle maintains a ballistic coefficient similar to MSL (less than 150 $\left.\mathrm{kg} / \mathrm{m}^{2}\right)$. 
The concept of operations is similar for the rigid Mid L/D, ADEPT and HIAD vehicles. All vehicles perform aerocapture with an arrival velocity of $6.2 \mathrm{~km} / \mathrm{s}$ and capture into a 1-sol parking orbit. The vehicle may loiter there for up to 1 year. Then EDL is initiated at the apoapsis of the parking orbit. As a risk mitigation approach during entry, the supersonic vehicle transition event of DRA5 and EDLSA has been eliminated, and with it the free-fall assumption associated with separating the lander from the entry vehicle. While this assumption simplifies EDL and offers protection for the payload from powered descent engine and surface plume interactions during landing, it complicates engine initiation and surface payload offloading for all the vehicle concepts. Refinement of subsystem interfaces continues and analyses are still being performed to determine an optimal method for exposing the engines at supersonic speeds for a vehicle that retains a large rigid heatshield. Likewise, analysis is also in progress to evaluate payload bay doors on the rigid Mid L/D vehicle to expose the payload after landing, what to do with the deployed ADEPT on the surface, and the details of how to retract the deflated tori of the HIAD after landing.

All architectures assume the use of supersonic retropropulsion to slow the vehicle for descent and landing. Additional drag devices like parachutes or trailing ballutes have been shown to have limited utility for EDL vehicles of this scale and are not included in this study. Other ground rules for EDL include maintaining a constant velocity of $2.5 \mathrm{~m} / \mathrm{s}$ for 5 seconds prior to landing at $0 \mathrm{~km}$ relative to the Mars Orbiter Laser Altimeter (MOLA) ellipsoid (nominally at the equator), within $50 \mathrm{~m}$ radius of a given target.

Finally, the aerodynamic decelerator, descent stage, and MAV payload are incorporated with the SEP stage in the launch vehicle fairing. The launch vehicle stack configuration for three architectures are shown in Figure 3.

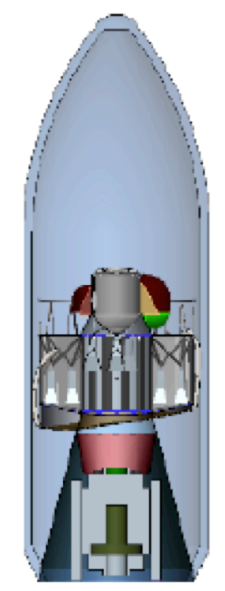

(a) ADEPT

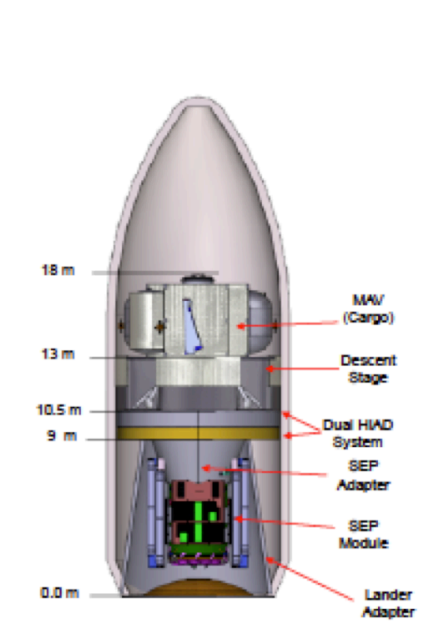

(b) HIAD

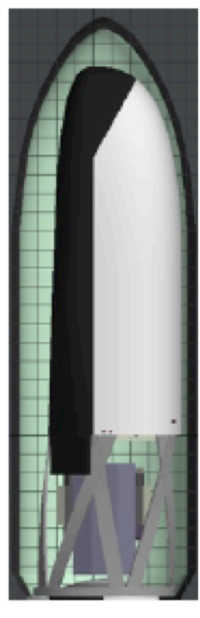

(c) Mid L/D

Figure 3. Launch vehicle stack configuration for (a) ADEPT, (b) HIAD and (c) Mid L/D.

\section{Approach}

To inform STMD EDL investment decisions, EDLAS used a two-part approach for the evaluation of technology performance. First, detailed lander point designs were created around the EMC project requirements for the payload and SLS payload fairing. This approach promoted the development and refinement of subsystem interfaces and the consideration for ground operations. The second approach used the point design solutions to anchor parametric models. Parametric models enable the vehicle to be scaled according to various input parameters. This broader view of the integrated architectures examines the extensibility of a technology to a range of parameters, such as ballistic number or entry velocity. Both approaches are described in this section.

Developing the detailed point designs required input from four subsystem teams. The first was the structural and packaging subsystem team. This team considered the total vehicle packaging arrangement and integration with the payloads (MAV and ISRU), the launch shroud, the descent stage and the entry technology. The team worked directly with each entry technology developer to adequately integrate the technology into the larger system. An initial mass estimate for the integrated lander was provided to the flight mechanics subsystem team to evaluate the nominal vehicle performance using trajectory simulations. The trajectory information was provided to the aerodynamics and aerothermodynamics team to perform high fidelity CFD and thermal analysis of each 
configuration. The resulting heat load and heat rate information was provided to the Thermal Protection System (TPS) subsystem team to select the TPS required to survive both aerocapture and entry heat pulses. The simulation results from the flight mechanics team provided an estimate of the vehicle loads and the propellant mass to the structural and packaging team for successive iterations.

Several factors contributed to the success of the integrated design approach. The first was that the EDLAS structures team was familiar with the EMC requirements and history of packaging challenges. They were aware of the launch vehicle shroud limitations and constraints and were able to facilitate much of the vehicle component integration. Another factor that advanced each point design was the use of focused design weeks. Members from each subsystem team were available to work through major subsystem integration challenges. Subsystem interfaces were redesigned until the design closed. Thirdly, having the subteams work on all vehicle configurations helped to ensure that similar margins and analysis practices were being used across the designs. For example, the same trajectory simulation and environment dispersion were used to evaluate each architecture. Only vehicle specific parameters (e.g. mass properties and aerodynamics) were modified. Likewise, some subsystems like avionics and power were common across all architectures. The primary product from this activity was an integrated closed design, including Master Equipment List (MEL), that could deliver a 20 t payload to the surface of Mars. The results are described in section 5 .

A fifth subteam, the mass sizing and system integration team, worked with each individual technology team and the other subsystem teams to develop a mass model of the baseline design that could be scaled parametrically. An integrated system analysis was developed to link the various models required to size and analyze the entry system. To date only the HIAD parametric model has been released. ${ }^{11}$ The integrated system uses the Program to Optimize Simulated Trajectories II (POST2) to model the flight mechanics. The aerodynamic database is based on existing wind tunnel and computational fluid dynamic results. Arcjet test results are the basis for the TPS parametric model. The HIAD fabric-sizing model uses an analytical model anchored with test results. The rigid nose uses analytical models based on NASTRAN (finite-element analysis) and FIAT (TPS sizing tool). The tool is anchored using the results from the point design work. The development of parametric models for ADEPT and the Mid L/D vehicle is underway.

The primary advantage of parametric models is that they enable assessments of the extensibility of each technology beyond the point design. It enables the identification of trends and breakpoints, such as the entry conditions that require a shift in TPS materials or the impact of payload mass on overall system sizing. When the parametric models for all of the decelerator architectures are completed, comparisons between them can be made.

While developing point designs and parametric models for each architecture are very informative for identifying interface challenges and performance metrics, there are still elements of the mission design that are not captured, such as cost, schedule and subsystem Technology Readiness Level differences between the designs. These elements were not documented as part of this study.

\section{Figures of Merit}

Since a NASA human mission to Mars is still decades away and firm requirements for a human mission to Mars are yet to be defined, it seemed prudent to characterize each technology relative to integrated system performance strengths and weakness and breakpoints. At this point in the study, the full assessment is not complete, but the following figures of merit (FOMs) have been identified for evaluation.

Several types of FOMs are considered. The first are quantitative FOMs that can be measured using performance simulations. They are based on the performance simulation of the $20 \mathrm{t}$ detailed point design developed for each technology and include (1) total post aerocapture delta $\mathrm{V}$, (2) the system control authority to meet the $50 \mathrm{~m}$ target landing constraint as well as the propellant required to do so, total arrival mass, total entry mass and number of launches required to land the complete set of surface mission equipment.

Parametric modeling can also identify quantitative FOMs by identifying system break points, or the limitations on the system where efficiency or system performance no longer improve the capability to deliver payload efficiently. Examples of such break points include (a) the maximum useful payload mass that can efficiently be delivered by the Mid L/D vehicle; (b) the payload mass for which a HIAD or ADEPT start to look like a capsule (i.e. ballistic coefficient is such that the vehicle diameter approaches the rigid shell diameter and requires a backshell to protect the payload; (c) the arrival velocities that require a change the TPS.

A second set is qualitative FOMs that, while not quantifiable like the previous set of FOMs, offer insight into the strength and weakness trades of each configuration. These FOM's include considerations for packaging and volume limitations, payload scalability, Technology Readiness Level (TRL), development cost and schedule, vehicle system integration, and end-to-end mission operations including ground operations and cargo offloading at Mars. 
The results of the FOM analysis will drive the prioritization of future mission design and technology research, development, and testing efforts, and will also be used to prioritize STMD investments. As more information emerges about the specific objectives of the human scale missions, NASA will be able to select the technology that best fits the need.

\section{Results}

The predicted mass results for the detailed point designs are presented in Table 1. Three integrated architectures were evaluated; the capsule results are still pending. While launch vehicle and SEP integration was considered, the MEL only includes the surface payload (20 t MAV + ISRU plant), the entry technology, the descent stage and associated landing subsystems. Table 1 provides the predicted masses (basic masses plus dry mass growth allowances) for the major elements of each architecture. The distribution of dry mass elements for HIAD and ADEPT are quite similar. For comparison with the Mid L/D configuration at this summary level, categories 1.0 and 6.0 should be considered in combination $(13,619 \mathrm{~kg}, 14,405 \mathrm{~kg}$, and 18,898 $\mathrm{kg}$ for ADEPT, HIAD, and Mid L/D, respectively). This provides a much more meaningful comparison of the grouping of primary structure, secondary structure, TPS, landing gear, and other mechanisms across the three decelerator concepts.

Table 1. Predicted Point Design Mass (with Contingency).

\begin{tabular}{ll|rrr}
\hline Mass & Breakdown Structure $(\mathrm{kg})$ & ADEPT & HIAD & Mid L/D \\
\hline $1.0 \quad$ Structures & 4,961 & 4,961 & 14,257 \\
$2.0 \quad$ Propulsion & 4,957 & 4,899 & 5,263 \\
$3.0 \quad$ Power & 1,217 & 1,217 & 1,217 \\
$4.0 \quad$ Avionics & 328 & 328 & 328 \\
$5.0 \quad$ Thermal & 328 & 328 & 347 \\
$6.0 \quad$ Entry Technology & 8,658 & 9,444 & 4,641 \\
$7.0 \quad$ Cargo & 20,000 & 20,000 & 20,000 \\
Dry Mass & 40,449 & 41,177 & 46,053 \\
8.0 Non-Propelled Fluids & 966 & 966 & 966 \\
Inert Mass & 41,415 & 42,143 & 47,019 \\
9.0 Used Propellant & 14,655 & 11,668 & 14,363 \\
\multicolumn{2}{l}{ Total Stage Gross Mass } & $\mathbf{5 6 , 0 7 0}$ & $\mathbf{5 3 , 8 1 1}$ & $\mathbf{6 1 , 3 8 2}$ \\
\hline \multicolumn{4}{r}{}
\end{tabular}

The HIAD configuration, with two separate inflatable structures, is the lightest architecture. However, at this early phase of design, the ADEPT vehicle mass is well within the mass margin of error relative to HIAD. The HIAD and ADEPT configurations do not offer protection for payload during in-space transport like the enclosed Mid L/D and capsule design. There are concerns about heating on the payload during aerocapture and entry for an exposed payload, a risk that may require a significant amount of additional mass to mitigate. Additionally, TRL, schedule, and cost are not represented.

The point designs analyzed for this study do not represent a final flight design. As mentioned in Section 3, the initial point design for ADEPT considered an asymmetric vehicle. A cross section view is shown in Figure 4a. Several challenges were identified with this design. The ring around the base of the descent stage that provided the structural support for the asymmetric design presented a challenge for the engines at initiation. The engines on one side of the vehicle were inset undesirably far inside the vehicle. Risk for flow recirculation around in the descent stage was a concern. Additionally, during launch, this ADEPT concept elevates a ring around the payload to retract the ADEPT like an umbrella. To accommodate this ring, the height of the descent stage element had to be increased relative to the height of the HIAD descent stage. This ADEPT design offers challenges in the launch phase, as well, because access to the payload was restricted. It also increases the height of the center of gravity of the landed payload, raising concerns about landing stability and cargo offloading. During a subsequent ADEPT design iteration, the overall decelerator asymmetry was eliminated and a fixed tab was added to maintain the lift vector. The image of the modified design is shown in Figure $4 \mathrm{~b}$. All of the vehicles configurations continue to evolve. 


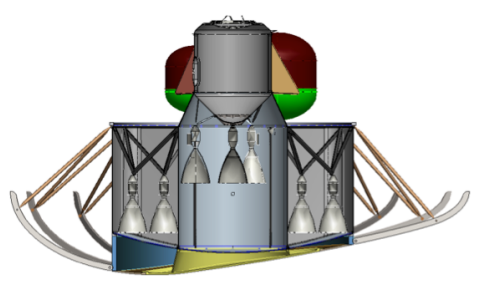

(a) Asymmetric

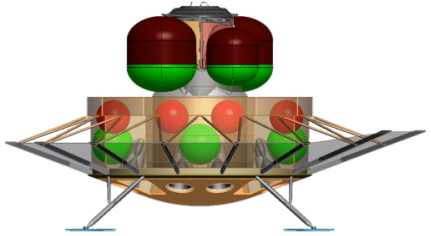

(b) Axisymmetric with fixed tab

Figure 4. ADEPT design progression.

In addition to developing point designs and parametric models, another objective of the study was to evaluate integrated system performance using trajectory simulations. One of the key areas of interest was vehicle controllability. Current state of the art is MSL, which jettisoned ballast masses to change the vehicle center of gravity to provide an L/D of 0.24 for entry. The vehicle used thrusters to perform bank angle modulation at a constant angle of attack to target a $19 \mathrm{~km}$ by $6 \mathrm{~km}$ ellipse in Gale crater, and jettisoned additional ballast mass to achieve zero angle of attach for parachute deployment. While this was an outstanding achievement over previous robotic landers, current EMC guidelines requires human scale landers to land within $50 \mathrm{~m}$ of a select target. Therefore, as a first analysis, bank angle guidance was paired with a pure gravity turn powered descent phase (meaning the vehicle follows the velocity vector). Several different variations of the algorithm were analyzed and all required more propellant (greater than $2 \mathrm{t}$ ) than the nominal case to meet the $50 \mathrm{~m}$ targeting constraint. Therefore, a second approach was used. In an effort to eliminate the open loop phase of bank angle guidance, a direct force control method was considered. This method uses direct angle of attack and sideslip forces (generated via a flap, movable mass or other device) to control vehicle attitude from entry through SRP engine initiation with lower angles of attack that reduce flow impingement on the payload. While work is still underway to characterize the controllability of all the configurations in all phase of flight, initial direct force control results indicate substantial savings in propellant over bank angle control for entry. However, the mass of the force control system and the associated impacts to vehicle subsystems, such as electrical power, must be assessed.

\section{Finding and Recommendations}

The integrated design approach for the individual point designs identified mismatched assumptions and system integration challenges associated with the concept of operations. This section highlights some of the findings and recommendations that have emerged from the study.

\section{A. Findings:}

- The EDLAS design process enabled a level of system analysis and understanding not previously achieved in EDL performance studies for this payload class.

- EDLAS was able to address nearly all of the major findings from the Deployable Decelerator Assessment. ${ }^{12}$

- The utilization of defined payloads (MAV, habitat, etc.) impacts the lander design and analysis. In previous design studies, the aerodynamic decelerators were parametrically scaled to maintain a specified ballistic number regardless of payload mass. Larger payload mass implied larger payload volume. For the MAV selected for this study, different propellant loading options can result in a range of payload masses for a fixed volume. Deployable entry systems cannot always be scaled to maintain a particular ballistic coefficient because of flow impingement concerns on the payload, and a lighter payload mass may not be associated a smaller/shorter payload as was assumed in previous studies. In addition, the Mid L/D configuration has additional constraints, since its drag area is not scalable independent of the dimensions available for payload packaging.

- Targeting the reduction of flow impingement rather than maintaining a fixed ballistic number may drive an increase in the diameter of the deployable decelerator technologies.

- For the HIAD and ADEPT configurations, the aerocapture and entry aerodynamic and aerothermal environments may require additional aft TPS to mitigate radiative heating on the exposed payload. Constraints on the aerocapture and entry flight profiles may also be required to avoid flow impingement/loads on the payload. 
- The Mid L/D vehicle is less mass efficient than the HIAD and ADEPT in terms of the ratio of launch (gross) mass to delivered payload on the surface of Mars. But the Mid L/D aeroshell fully protects the payload during aerocapture and entry.

- In the SLS launch configuration, the center of gravity of the Mid L/D vehicle will be substantially higher than the HIAD or ADEPT cg locations. This will reduce the modal frequency and increase the loads on the SLS upper stage.

- Engine performance and RCS propellant storage assumptions have significant impacts on the descent stage design. The Isp of the SRP main engines and RCS thrusters is a critical driver on the usable propellant mass. Zero boiloff is also essential for a cryogenic propulsion system employed on a long duration mission.

- Developing and sizing mechanisms for exposing engines and landing gear is a challenge common to all decelerator concepts and requires knowledge of the environment at engine initiation.

- The detailed design effort provided inertia and center of gravity location for each lander, which enabled detailed assessments by the flight mechanics subsystem team to determine requirements for vehicle controllability, terminal descent sensor and precision landing requirements.

- Bank angle guidance is not a propellant optimal approach to meet the $50 \mathrm{~m}$ diameter landing constraint for the HIAD and ADEPT vehicle.

- The EMC requirement for pinpoint landing substantially changes the guidance, navigation, and control paradigm relative to the large landing ellipses present on previous robotic missions to Mars.

- More detailed surface operations assessments are required for all decelerator concepts to address cargo offloading, crew access, and MAV launch operations.

\section{B. Recommendations:}

- Further guidance is needed regarding NASA's goals for human missions to Mars. Priorities such as Design Development Test and Evaluation (DDT\&E) or total cost and schedule may favor a different lander approach than prioritization on minimum mass or a reduced number of launches, for example.

- EDL technology downselect should not be performed until more is known about integrated Mars landing system performance and scalability and the parametric mass models are sufficiently developed to allow comparison of the designs.

- Supersonic separation of the payload from the lander aeroshell during the Mars EDL trajectory has not been evaluated to the extent that it can absolutely be ruled out of the lander design space. While the separation of a large aeroshell under significant aerodynamic loads is challenging, this approach requires less propellant and potentially simplifies Mars ground operations. However, supersonic separation of the aeroshell also increases heating concerns relative to the SRP flowfield and may also require significant EDL trajectory shaping and divert maneuvers to protect emplaced surface assets from debris impact while still achieving pinpoint landing.

\section{Conclusion}

This paper summarizes the Mars EDL Architecture Study's approach to informing STMD investments through Phase A level designs, parametric modeling and trajectory analysis. Because the study is ongoing, no recommendations have been made regarding the prioritization of the various aerodynamic decelerator technologies under consideration. This paper outlines how the EMC project requirements, like the use of aerocapture and surface delivery of a $20 \mathrm{t}$ MAV payload, flows down to the system (e.g., launch vehicle packaging) and subsystem level (e.g., determining optimal control methods). Through simulation and modeling the study assessed a wide range of lander design requirements, such as evaluating angles of attack during entry that reduce flow impingement on the exposed payload for HIAD and ADEPT. The study evaluated and refined key subsystem interface options, such as modifying the asymmetric ADEPT shape to an axisymmetric shape with a fixed tab after identifying complications with SRP engine placement and firing in an enclosed area behind the heat shield. Through component modeling, three complete system-level designs were presented. Parametric modeling anchored by these point designs will enable the team to assess breakpoints and, ultimately, identify the more favorable mission/system architectures.

This study has illuminated many of the challenges of integrated system design that become more apparent through higher fidelity analyses. Integrating the parametric models into the trajectory simulation allows for detailed analysis of variations in each design. The results can inform and guide future ground and flight tests. While the results of this study have not identified any specific showstoppers associated with the four aerodynamic decelerator 
technologies, additional analysis using the models and simulations developed during this study will be useful in quantifying the specific strengths and weakness and identify breakpoints for each configuration.

\section{Acknowledgements}

The authors would like to recognize the members of the EDLAS Team. They come from many NASA centers including Ames Research Center, Langley Research Center, Johnson Spaceflight Center and Marshal Space Flight Center. While the individuals involved are too numerous to list here, the work summarized in this paper is based on their collective contributions.

\section{References}

${ }^{1}$ Craig, Douglas A., Herrmann, Nicole B., and Troutman, Patrick A. “The Evolvable Mars Campaign - Study Status,” IEEE Aerospace Conference, Big Sky, MT, March 7-14, 2015.

${ }^{2}$ Polsgrove, Tara P., et al. "Mars Ascent Vehicle Design for Human Exploration.” AIAA SPACE2016 Long Beach, CA September 13-16.

${ }^{3}$ NASA Space Flight Program and Project Management Handbook NASA/SP-2014-3705.

${ }^{4}$ Drake, B. G., editor, "Human Exploration of Mars Design Reference Architecture 5.0,” s.1. : NASA SP-2009-566, 2009.

${ }^{5}$ Dwyer Cianciolo, A. M., et al., "Entry, Descent and Landing Systems Analysis Study: Phase 1 Report," NASA-TM-2010$216720,2010$.

${ }^{6}$ Cerimele, C., Robertson, E., Sostaric, R., Campbell, C., Robinson, P., Hershey, M., Stachowiak, S., Matz, D., Braden, E., Johnson, B., Garcia, J., Bowles, J., Kinney, D. “A Rigid Mid Lift-to-Drag Ratio Approach to Human Mars Entry, Descent, and Landing” AIAA SciTech 2017 Grapevine TX (Abstract submitted).

${ }^{7}$ Price, H., et al. "A High-Heritage Blunt-Body Entry Descent, and Landing Concept for Human Mars Exploration” AIAA2016-0219.

${ }^{8}$ Cassell, A., et al "Human Mars Mission Design Study Utilizing the Adaptive Deployable Entry and Placement Technology," IEEE Aerospace Conference, Big Sky, MT 2017 (abstract submitted).

${ }^{9}$ Hughes, S. J., et al. "Hypersonic Inflatable Aerodynamic Decelerator (HIAD) Technology Development Overview," 2013

${ }^{10}$ Polsgrove, Tara P., Dwyer Cianciolo, Alicia, et al., "Human Mars Lander Design for NASA's Evolvable Mars Campaign," IEEE Aerospace Conference, Big Sky, MT, March 5-12, 2016.

${ }^{11}$ Samareh, J., Dutta, S., Cianciolo, A. D., Komar, D. R. "Rapid Systems Analysis and Trade Studies for Human Mars EDL" IEEE Aerospace Conference, Big Sky, MT 2017 (abstract submitted).

${ }^{12}$ Corliss, J., et al. "Deployable Decelerator Assessment” Nov. 2013. NASA internal study. Unpublished. 